\title{
Controlling a variable geometry truss tetrobot with an isomorphic puppet controller
}

\author{
Robert L. Read \\ Public Invention President, 1709 Norris Dr, Austin, TX \\ 78704, +1 5129136130 \\ read.robert@gmail.com
}

\author{
Avinash Baskaran \\ Graduate Researcher, Wearable and Bio-Robotics Lab, 354 \\ War Eagle Way, Auburn, AL 36849, +1 9725230190 \\ baskaran.avinash@gmail.com
}

\begin{abstract}
Humans are skillful. By building a bio-inspired manipulable snakelike controller that can be molded into a wide variety of shapes, we allow a human controller to telepresently specify complex shapes and shape changes. We constructed a tetrahelix consisting of seven tetrahedra made of adjustable-length members connected via 3D printed Song-Kwon-Kim joints which allow manual changes to the shape of the controller. These changes in length are digitized and organized via an Arduino and transmitted to more powerful computers where they may specify a shape to be animated or control a robot of similar shape, or simply specify relative positions in Cartesian space. Using the controller it is easy to control an isomorphic, but larger, 7-tet tetrobot, touch a target with an end effector, and pick up a weight with a hook. The controller allowed gait design that would otherwise have been very tedious. Although this research is basic, we hope it will eventually amplify human control of in vivo mechanical devices such as endoscopes, searchand-rescue robots weaseling into tight spaces, or general purpose tetrobots used for planetary space exploration as suggested by Prof. Sanderson and his students 20 years ago.
\end{abstract}

\section{CCS CONCEPTS}

- Human-centered computing $\rightarrow$ Human computer interaction (HCI); Interaction devices.

\section{KEYWORDS}

Robots, Variable Geometry Truss, Telepresence, Tetrahelix, Snakelike robots

\section{ACM Reference Format:}

Robert L. Read and Avinash Baskaran. 2020. Controlling a variable geometry truss tetrobot with an isomorphic puppet controller. In $20202 n d$ International Conference on Artificial Intelligence, Robotics and Control (AIRC'20), December 12-14, 2020, Cairo, MN, Egypt. ACM, New York, NY, USA, 5 pages. https: //doi.org/10.1145/3448326.3448332

\section{INTRODUCTION}

The possibility of building a robot based on tetrahedra constructed of tensegrities has been well researched by Hamlin and Sanderson [1], NTRT [2], Paul et al. [3], and Chen et al. [4]. It is possible to construct a variable geometry truss out of actuators using tetrahedra as

CC.
This work is licensed under a Creative Commons Attribution International
4.0 License.
AIRC'20, December 12-14, 2020, Cairo, MN, Egypt
2020. ACM ISBN 978-1-4503-8926-6/20/12.
https://doi.org/10.1145/3448326.3448332

a repeated module that might be expected to have an advantageous strength-to-weight ratio.

The existence of the Boerdijk-Coxeter tetrahelix [5-8] has long been recognized as a geometric means of composing tetrahedra into long beams, which might combine the structural advantages of a tetrahedral tensegrity with snake-like robot motion $[9,10]$.

By utilizing a 3D-printed jointing system which supports angular displacement of multiple members coming to a central point extending previously patented work [11] and small-scale actuators and microcontrollers, the authors have constructed a relatively inexpensive tetrobot. Although some headway has been made in numerical control, this paper explores the fundamental idea of using a simulacrum controller. A controller which is isomorphic to the robot (but smaller) is constructed which can be manipulated by hand by an operator. The larger robot mimics the motion of the controller. This controller, called the tetrocon, has been used to develop a better hexapodal walking and turning gaits than previously possible for the tetrobot. Striking an object in space with the end effector is easy with the controller. The controller even allows, with some effort, locomotion around or over obstacles. This paper reports on the tetrocon and its usage.

\section{MOTIVATION}

Although developed to control the tetrobot, the controller system we propose is versatile, and can be used independently as a shapeinput device. Applications include biomimetic robotics and animatronics, telepresent control of basically snake-like or tentacle-like endoscopic or arthroscopic surgical robots and terrestrial locomotion, reported on below.

\section{DESIGN AND MANUFACTURE}

All hardware and software designs of the controller are free-libre open source [12].

A tensegrity is a device in which each member only supports tensile or compressive loads or both. No member must resist angular displacement. Tensegrities usually use cables for the tensile components and rods for the compressive components. Tensegrity robots vary the length of one or the other. Since a cable attached to a point can pull in any direction, the tensegrity condition is achieved by attaching rods only to cables, not other rods.

However, Hamlin and Sanderson [13] produced a novel concentric multilink spherical (CMS) joint based on parallelograms. Such joints allow the construction of tensegrities without cables (or, equivalently, where the cable length has shrunk to zero.) 


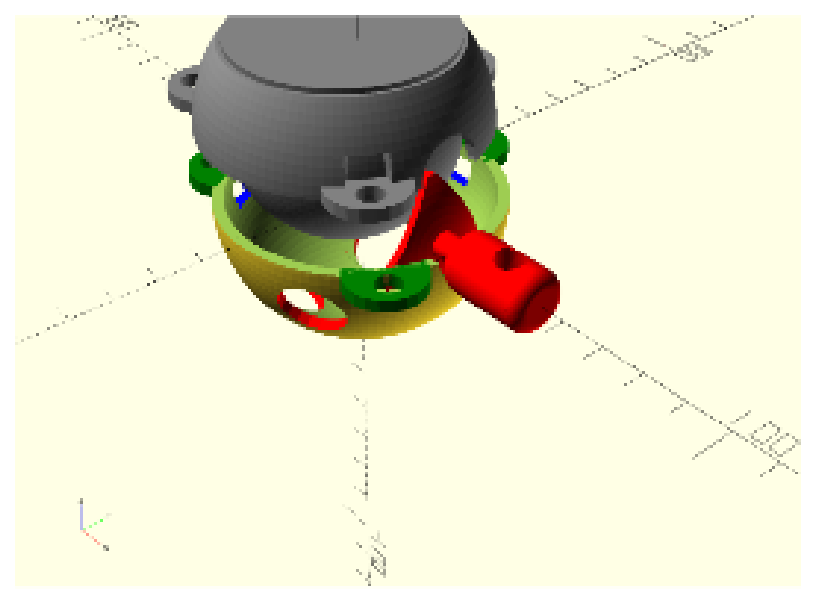

Figure 1: Exploded view of Song-Kwon-Kim joint

\subsection{The Song-Kwon-Kim Concentric Multimember Joints}

Somewhat later Song, Kwon and Kim [11] patented a modification of a ball-and-socket joint which is roughly similar. (See Fig. 1) 3D printing makes the construction of the Song-Kwon-Kim joint practical and inexpensive, though it is not necessarily mechanically superior to the CMS joint.

Such joints allow multiple rods to connect around a single point with spherical rotation, automatically forming a tensegrity.If those rods can change length on their own power, they form a machine or robot. If those rods can be changed by external forces, they form an Input device. If you build both, you can have a robot, such as is pictured in Fig. 5, which mimics a simulacrum or puppet controller you can shape with your hands, such as in Fig. 4

\subsection{The Lock Component Designed for a Tetrahelix}

In a Song-Kwon-Kim style joint, a spherical lock holds rotors in place. The placement of the holes for the rotors depends on the geometry of the robot one is trying to create. For tetrahedral tensegrities wherein the ratio of the maximum length of an actuator to the minimum does not exceed the golden ratio, the holes can be placed so that all configurations of a single tetrahedron are possible.

An improvement of the Song-Kwon-Kim joint is to make the rotor triangular. Triangular rotors allow for the expansion of the opening from which the shaft penetrates, allowing greater angular displacement of the shaft. So long as the rotors can revolve, then in the tighter configuration the rotors meet edge-to-edge. This has the further advantage of strengthening the points of contact with the lock when in tension.

\subsection{A Universal Rotor}

Early prototypes of our version of the joint connected the actuator to a rotor in a way that was difficult to disconnect. We have replaced this with a standard, or universal, rotor shape. The universal rotor

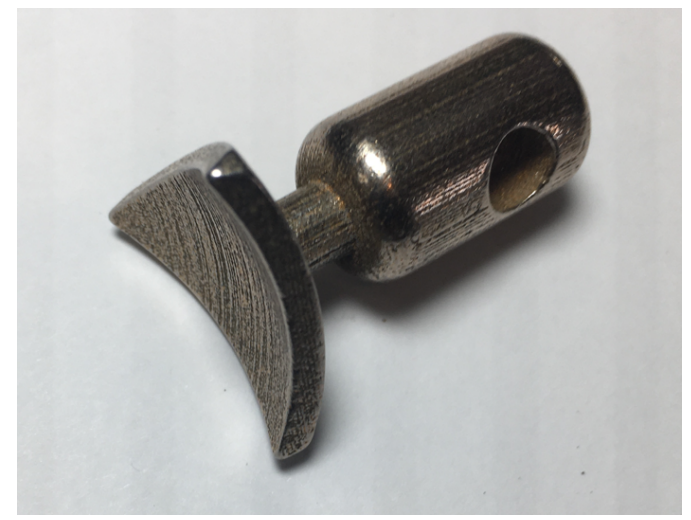

Figure 2: A 3D-printable steel rotor

has a hole which allows it to be detached from the sensor or actuator connected to it by removing a zip tie or a bolt.

The result is that the joint itself does not have to be taken apart to repair an actuator or displacement sensor. This has been a great help in the maintenance of the robot.

In our construction of the robot, we found it necessary to make some pieces of the joint out of metal (Fig. 2), whereas with the smaller scale and weaker forces of the controller the same parts were serviceable when $3 \mathrm{D}$ printed in plastic.

\subsection{The Controller}

The tetrocon (see Fig. 4) device also uses 3D printed Song-KwonKim joints. The holes in the locking shell of this joint are centered to mimic a tetrahelix (slight modifications of this configuration would be needed for other geometries.)

Linear potentiometers serve as linear displacement sensors. These sensors are held in snap-together 3D printed sleeves, which have female parts to receive our universal jointing system. The entire system is modular, in the sense that every joint and every member is precisely the same. It snaps apart easily but is easy to repair.

Because the controller is meant to be moved by hand through its range of operation with minimal forces, we have found 3D-printed plastic parts acceptable, although occasionally we have a layer separation break with our rotors.

Electronically, each of six potentiometers (forming a twotetrahedra modular extension) is connected to a multiplexer, which controls which signal is sent to an Arduino Uno microcontroller. This allows the 24 displacement sensors in a 7-tetrahedron controller to be digitized. A simple program returns all values upon request in JSON. A Python program implements a web server, which allows other software to query the state of the 24 channels in the controller simply by making a web request. The authors have produced an interactive virtual model of the Tetrocon which is available online [13] and is pictured in Fig. 3

\subsection{The 7-Tet Tetrobot}

The 7-Tet Tetrobot (see Fig. 5), hereinafter simply called the tetrobot, uses electromechanical actuators with a long lead screw driven by 


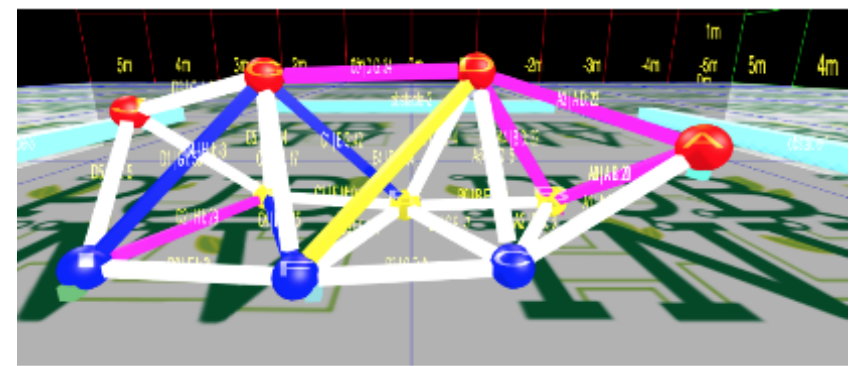

Figure 3: Live virtual model of Tetrocon in webpage [13]

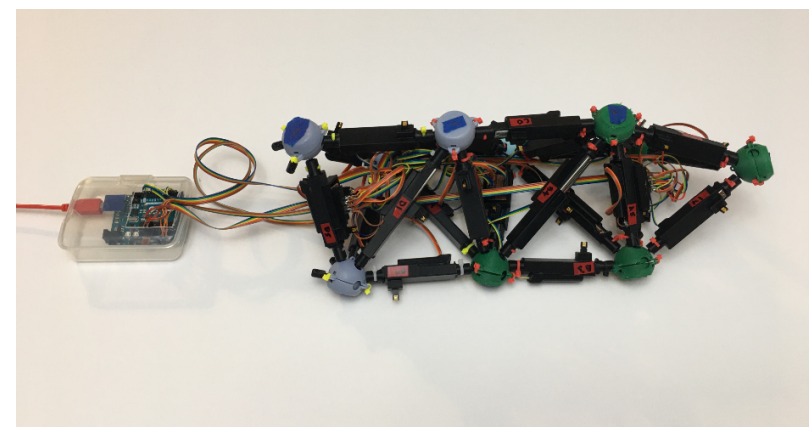

Figure 4: Tetrocon hand-manipulable puppet controller

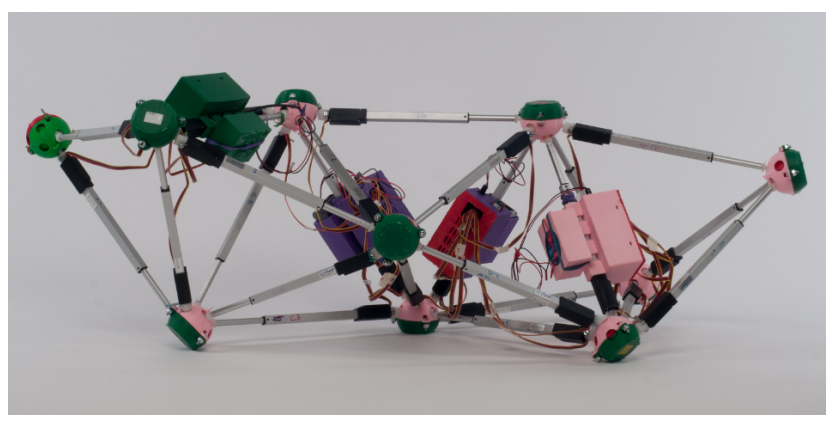

Figure 5: The 7-tet tetrobot robot

a small rotary motor. They can exert 50 Newtons (11 pounds) of force, while weighing only a few hundred grams.

The tetrobot consists of four independent modules, each having (and carrying) a $12 \mathrm{~V}$ NiMH battery, by far the heaviest component of the robot. Thus, in theory the tetrobot is expandable to any number of modules while remaining untethered.

Each module controls six actuators, so the addition of a new module adds two more tetrahedra to the system. Each module has a Bluetooth radio on a custom Arduino Mega shield, along with three two-channel motor controllers. The Arduino runs code which accepts commands to change the length of members via Bluetooth. These commands are submitted by an Emacs electric lisp programmer, making it convenient for an operator to issue commands in an Emacs lisp buffer. The 7-Tet Tetrobot is thus an untethered locomotive (crawling) robot.
The case to hold the battery and controller for each module is $3 \mathrm{D}$ printed with an orifice allowing it to be mounted on the fixed part of an actuator.

In the tetrobot, the universal rotor is composed of $3 \mathrm{D}$ printed steel fabricated by Shapeways (Fig. 2). Each end of an actuator is bolted to a universal rotor. We attempted to attach the pushrod to the universal rotor with a 3D printed plastic part but found it necessary to move to an aluminium part. A local machinist made the threaded pushrod connectors for us. The other end of the actuator is larger and less prone to buckling forces, and we have so far been successful with plastic parts there.

The shell and ball of the Song-Kwon-Kim joint in fact is under very little force and 3D printed plastic parts have been acceptable. However, our current design requires the shell to be bolted to a cap, and the bolts are prominent. When on the ground, they tend to catch and produce uneven forces. We intend to move to a low-profile design soon.

\section{USE OF CONTROLLER WITH TETROBOT}

\subsection{End-effector positioning}

The simplest task for any such robot is end-effector positioning in space. By orienting the tetrocon to match the physical position of the tetrobot, volunteers from an audience with zero experience can easily cause the first joint of the tetrobots to hit a positioned object after a few seconds of orientation. In a sense, this is a simulation of a robot "arm" that does not support locomotion.

\subsection{Locomotion}

A variable geometry truss wherein each element can change length has high input dimensionality; the 7-tet tetrobot has 24 actuators. The authors have previously explored developing gaits using a virtual model of the tetrobot [16]. This system used the ammo physics engine to simulate gravity. The basic goal is to position six joints on the ground and then move one joint at a time, akin to a hexapodal gait on a robot with legs. Although some progress was made, the complexity of the software and physics simulation had many limitations.

The controller, operating without the tetrobot, allowed us in a single hour to develop a set of thirteen positions which allows the robot to move forward on a smooth surface fairly effectively. When we were able to use the tetrobot with the tetrocon controller and see the effect of the robot position change directly within one or two seconds of changing the controller, we found the two together made it much easier to develop motions and gaits for the robot. We discovered a more efficient "inchworming" gait which proved practical with two hours of experimentation. When recorded and replayed, this gait allows the tetrobot to move up to $0.38 \mathrm{~cm} / \mathrm{s}$, as shown in Table 1. Gait Speeds.

Although the Tripod gait is expected to be faster, we discovered that its multiple tetrahedra can exceed the angular limit of our joint. Improving the joint or providing software limitations to prevent this are the highest priority future work.

The controller allowed the creation of a set of poses that accomplished a $45\left\{^{\circ}\right\}$ turn with the same ease (see Table 2). The tetrobot turns about $45\left\{^{\circ}\right\}$ in about 44 seconds. 
Table 1: Gait speeds

\begin{tabular}{lcccc}
\hline \multicolumn{1}{c}{ Gait } & Trial & Distance & Time & $\begin{array}{c}\text { Average } \\
\text { speed }\end{array}$ \\
\hline Inchworm & 0 & $12.5 \mathrm{~cm}$ & $32.99 \mathrm{~s}$ & $0.38 \mathrm{~cm} / \mathrm{s}$ \\
& 1 & $6 \mathrm{~cm}$ & $55.04 \mathrm{~s}$ & $0.11 \mathrm{~cm} / \mathrm{s}$ \\
Conwalk & 2 & $11 \mathrm{~cm}$ & $33.1 \mathrm{~s}$ & $0.33 \mathrm{~cm} / \mathrm{s}$ \\
& 0 & $10 \mathrm{~cm}$ & $57.65 \mathrm{~s}$ & $0.17 \mathrm{~cm} / \mathrm{s}$ \\
Tripod & 1 & $12 \mathrm{~cm}$ & $55.14 \mathrm{~s}$ & $0.22 \mathrm{~cm} / \mathrm{s}$ \\
& 2 & $6 \mathrm{~cm}$ & $54.43 \mathrm{~s}$ & $0.11 \mathrm{~cm} / \mathrm{s}$ \\
& 1 & $6.5 \mathrm{~cm}$ & $16.5 \mathrm{~s}$ & $0.39 \mathrm{~cm} / \mathrm{s}$ \\
& 2 & $9 \mathrm{~cm}$ & $16.85 \mathrm{~s}$ & $0.53 \mathrm{~cm} / \mathrm{s}$ \\
\hline
\end{tabular}

Table 2: Turning speeds

\begin{tabular}{ccc}
\hline Trial & Angular displacement & Time \\
\hline 0 & 44 & $44 \mathrm{~s}$ \\
1 & 42 & $44 \mathrm{~s}$ \\
2 & 45 & $53 \mathrm{~s}$ \\
\hline
\end{tabular}

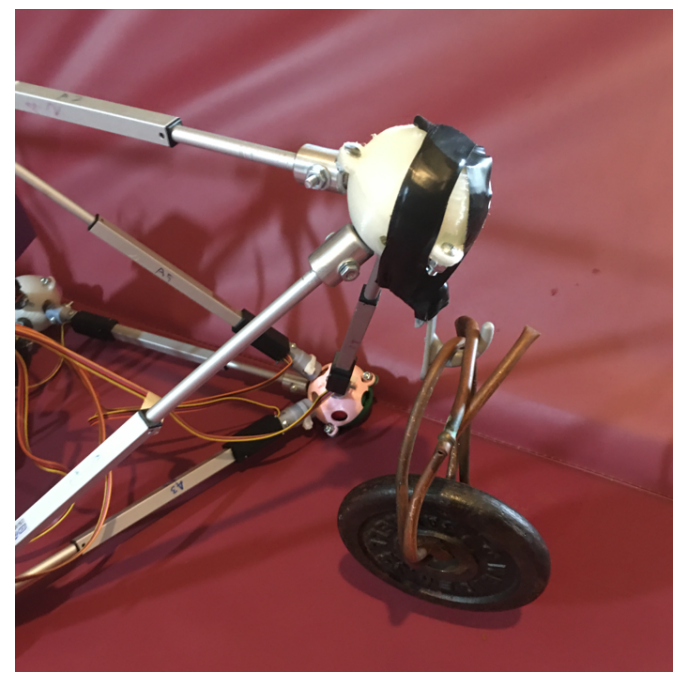

Figure 6: Side view of Tetrobot lifting 3-pound iron weight

\section{MANIPULATION}

The point of the Tetrobot is to make a general-purpose machine. Unlike wheeled robots which may have a manipulator attached to the locomotive chassis, the Tetrobot is a manipulator as well as a locomotor.

Using the Tetrocon and taping a hook onto the "head" node, it was relatively easy for an operator to pick up and lift a 3 pound weight (see Figs. 6 and 7). Without the Tetrocon controller, this might have been a very difficult to program. The Tetrobot only weighs 15 pounds with its batteries, but can even walk forward carrying this weight, although it tends to fall off the hook.

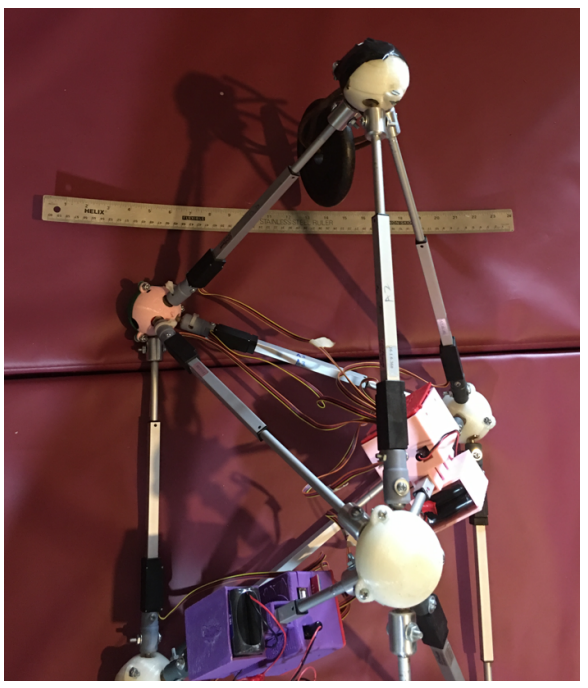

Figure 7: Top view of Tetrobot lifting 3-pound iron weight with 24 " ruler

\section{FUTURE WORK: VIRTUAL CONTROL}

Because the shape of a tetrahedron is completely determined by the length of its sides, it is possible to completely reconstruct the shape of the controller from its input. Software to do so is available on our site [13]. Improving the joint to allow complete flexibility of any tetrahelical configuration is the highest priority.

Because a tetrahelix $[5,8]$ is basically cylindrical or tentacle-like in shape, one can use the tetrocon to define a wide variety of shapes in space. In fact the tetrocon supports a certain amount of size or thickness information as well. However, if valuable, you could consider only the centroid of each tetrahedron and thereby have a hand-manipulated specifier of curves in 3D Cartesian space.

Thus any robot which is somewhat tentacle-like in shape $[15,16]$ could be controlled by the tetrocon. Since the tetrocon is modular, the length, in terms of the number of tetrahedra, is essentially unlimited. In practice, it becomes difficult to hold an object with too many tetrahedra. Our controller currently suffers from not offering enough resistance to change in displacement. That is, it is easy to move it into any position with the hands, but the potentiometers slide so easily that gravity may pull it back to the table or cause it to sag between a user's hands.

\section{CONCLUSION}

The use of a hand-manipulable controller that is the same shape as the controlled robot allowed a complex tetrobot to be effectively telepresently controlled to develop programmable gaits and allowed a human robot pilot to lift objects with relative ease.

\section{REFERENCES}

[1] Hamlin, G. J., Sanderson, A. C., Springer Science and Business Media, Tetrobot: A Modular Approach to Reconfigurable Parallel Robotics, (2013)

[2] NTRT - NASA Tensegrity Robotics Toolkit, https://ti.arc.nasa.gov/tech/asr/ groups/intelligent-robotics/tensegrity/NTRT/ (2016)

[3] Paul, C., Valero-Cuevas, F.J., Lipson, H., Design and Control of Tensegrity Robots for Locomotion. IEEE Transactions on Robotics, 22(5), (2006). 
[4] Chen, L., Kim, K., Tang, E., Li, House K., R., Zhu. E. L., Fountain, K., Agogino ,A M., Sunspiral, A, V J. "Soft spherical tensegrity robot design using rod-centered actuation and control”, Journal of Mechanisms and Robotics, 9(2);025001, (2017)

[5] Coxeter, H. S. M. et al, "The simplical helix and the equation tannt=ntant." Canad. Math. Bull, 28(4), 385-393 (1985)

[6] Fuller, R. B., Applewhite, E. J., Synergetics: explorations in the geometry of thinking, Macmillan, ISBN: 9780025418707 (1982)

[7] Gray, R. W., Tetrahelix Data, access online at: https://www.rwgrayprojects.com/ rbfnotes/helix/helix0.html (2017)

[8] Read, R. L., Transforming Optimal Tetrahelices Between the Boerdijk-Coxeter Helix and a Planar-Faced Tetrahelix. Journal of Mechanisms and Robotics 10, no. 5 (2018).

[9] Hirose, S., Biologically inspired robots: snake-like locomotors and manipulators. Oxford Science Publications (1993)
[10] Liljebäck P., Pettersen, K. Y., Stavdhal, O., Gravdahl, J. T., Snake Robots: Modeling, Mechatronics and Control (2012)

[11] Song, S. K., Kwon, D. S., Kim, W. S., US Patent : 6,568,871 : Spherical joint for coupling three or more links together at one point, (2003)

[12] GlussCon. https://github.com/PubInv/GlussCon (2020)

[13] TetroBot. http://pubinv.github.io/tetrobot/playground (2020)

[14] Hamlin, G. J., Sanderson, A. C., "A novel concentric multilink spherical joint with parallel robotics applications", ICRA, 2, 1267-1272 (1994)

[15] Mikulas, M. M., Crawford, R. F., US Patent 4,557,097: Sequentially Deployable Maneuverable Tetrahedral Beam (1985)

[16] Mirletz, B. T., Park, I., Flemons, T. E., Agogino, A. K., Quinn, R. D., Sunspiral, V., "Design and Control of Modular Spine-Like Tensegrity Structures" IACSM, 41 (2014) 